\title{
Charm production cross section at CDF II
}

\author{
Elena Gramellini and Manuel Mussini* \\ On behalf of the CDF collaboration \\ INFN and University of Bologna, Viale Berti Pichat 6/2, 40127, Bologna, Italy \\ E-mail: elena1987@gmail.com, manuel.mussini@gmail.com
}

In the last decade, the CDF experiment at the Tevatron collider clearly demonstrated that it is possible to study heavy-flavor physics extensively in hadron-hadron colliders collisions and achieve remarkable results, competitive and complementary to B-factories and LHC. In this paper, we report on the differential production cross-section measurements of $D$ mesons $\left(D^{0}\right.$ and $\left.D^{ \pm}\right)$extended to low transverse momentum. This measurement probes a region where perturbative $\mathrm{QCD}$ (pQCD) models are not able to describe the heavy-quark production because of the running of the strong coupling constant, $\alpha_{S}$. The data samples collected at two different center of mass energies, $1.96 \mathrm{TeV}$ and $900 \mathrm{GeV}$, also give the opportunity to assess the energy dependence of the production cross section.

14th International Conference on B-Physics at Hadron Machines April 8-12, 2013

Bologna, Italy

* Speaker. 


\section{Introduction}

The early CDF measurement of the prompt charm production cross section [1] had a significant impact in the QCD community. It was the first measurement of charm production in hadron-hadron collisions in a scenario where large discrepancies were observed between measured heavy-flavor cross sections and NLO predictions. However, the kinematic regime probed by that measurement was limited, since the cross section, differential in the $D$-meson transverse momenta, probed a minimum $p_{T}$ of $5.5 \mathrm{GeV} / \mathrm{c}$, because of the biases introduced by the trigger selection. Clearly, extending the measurement to lower transverse momenta would be extremely useful for the theory, providing additional experimental lever arm to refine the calculations in a regime where $c$ quark production is in non-perturbative conditions [2]. The huge amount of data collected during CDF Run II gives the chance to study the production of heavy-flavored mesons in the minimum-bias (MB) sample, which is the only one where it is possible to extend the transverse-momentum range of the produced particles to the lowest value achievable at CDF. By construction, the MB trigger has minimal and very generic requirements in order to reduce any kind of biases to the physics of the collected data but, of course, it carries the penalty of a very small quantity of heavy-flavor events in the huge light-quark background.

Here we report the attempt of reconstructing a charm signal in the MB sample in the channels $D^{0} \rightarrow K^{-} \pi^{+}$and $D^{+} \rightarrow K^{-} \pi^{+} \pi^{+}$. In what follows C-conjugate decays are implied and branching fractions are $\mathrm{CP}$ averages.

\section{Analysis overview}

The $D^{0} \rightarrow K^{-} \pi^{+}$and $D^{+} \rightarrow K^{-} \pi^{+} \pi^{+}$channels are the simplest charm topologies that can be studied at CDF II to detect these two charmed mesons, because they have a relatively high branching ratio (about $3.9 \%$ for the $D^{0}$ and about $9.1 \%$ for the $D^{+}$) and they can be fully detected by the tracking system.

To unfold the $D^{0}\left(D^{+}\right)$signal from the background the track helices are fitted looking for an intersection point displaced from the production point (primary vertex). If the fit returns a possible common origin for the two (three) tracks, a $D^{0}\left(D^{+}\right)$"candidate" is found.

Studying the decay topologies is fundamental to decide the selection variables in order to optimize the signal significance. Figure 1 shows some of the fundamental quantities used in this analysis for both the channels studied:

- $\vec{p}_{T}$, the transverse momentum, is the projection of the momentum vector to the plane transverse to the beam line.

- $L_{x y}$, the transverse decay length, is the signed distance between the primary and the secondary vertices projected to the transverse plane. It is defined as follows:

$$
L_{x y}=\frac{\left(\vec{x}_{s e c}-\vec{x}_{p r i}\right) \cdot \vec{p}_{T}}{p_{T}}
$$

where $\vec{x}_{p r i}$ is the projected position of the $p \bar{p}$ collision and $\vec{x}_{s e c}$ is the projected position of the decay vertex. 

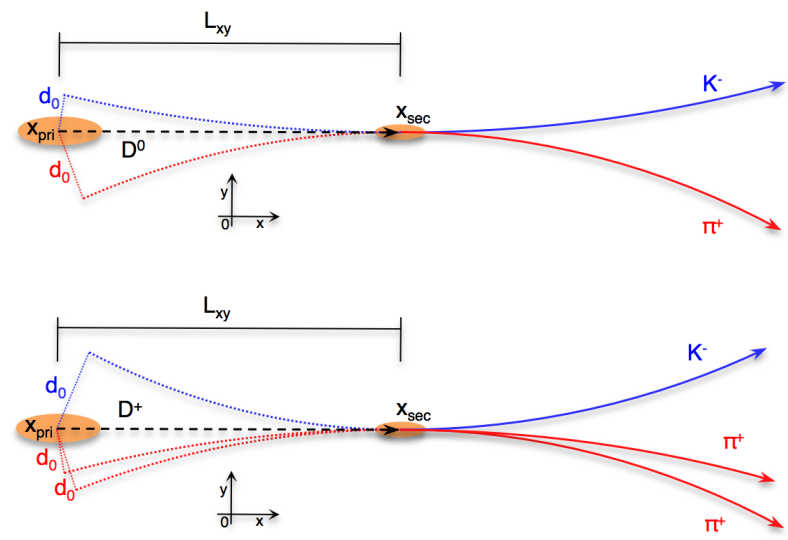

Figure 1: Scheme of the topologies of the $D^{0}$ and $D^{+}$decay channels in the transverse plane.

- $d_{0}$, the impact parameter, is the signed distance between the primary vertex and the track helix at their point of closest approach.

We optimize the selection cuts directly on data maximizing the figure of merit in Equation 2.2

$$
F(S ; B)=\frac{S}{\sqrt{S+B}}
$$

where $\mathrm{S}$ and $\mathrm{B}$ are the fitted signal and background yields in the region of the $D^{0}$ peak (1.864 $\mathrm{GeV} / \mathrm{c}^{2} \pm 2 \sigma_{m_{D^{0}}}$ ). To get the (unbiased) optimized invariant mass plots shown in the following sections, we use the following procedure:

- We divide the sample into two random subsamples (e.g., even and odd events).

- We optimize the selection separately for each of the subsamples.

- We resum the two subsamples applying the optimized selection of the former to the latter and viceversa.

\section{Cross section measurement}

\section{1 $D^{0}$}

We look for candidates fitting any possible combination of two reconstructed tracks with opposite charge in each event, assuming that the negative ones are due to a $K^{-}$and the positive ones to a $\pi^{+}$. We then plot the candidate's invariant mass and we fit the signal yield. In this $p_{T}$ range the particle ID is not available, so our signal is composed of both $D^{0}$ and $\bar{D}^{0}$ candidates. Figure 2 shows the invariant $K^{-} \pi^{+}$mass distribution at both $\sqrt{s}=1.96 \mathrm{TeV}$ and $\sqrt{s}=900 \mathrm{GeV}$ of all the candidates with $p_{T} \geq 1.5 \mathrm{GeV} / \mathrm{c}$. Figure 3 shows the measured yields ${ }^{1}$ as a function of $p_{T}$.

\footnotetext{
${ }^{1}$ The candidate selection is optimized independenlty for each bin; this explains why the sum of the yields of Figure 3 is different from the candidates fitted in Figure 2.
} 

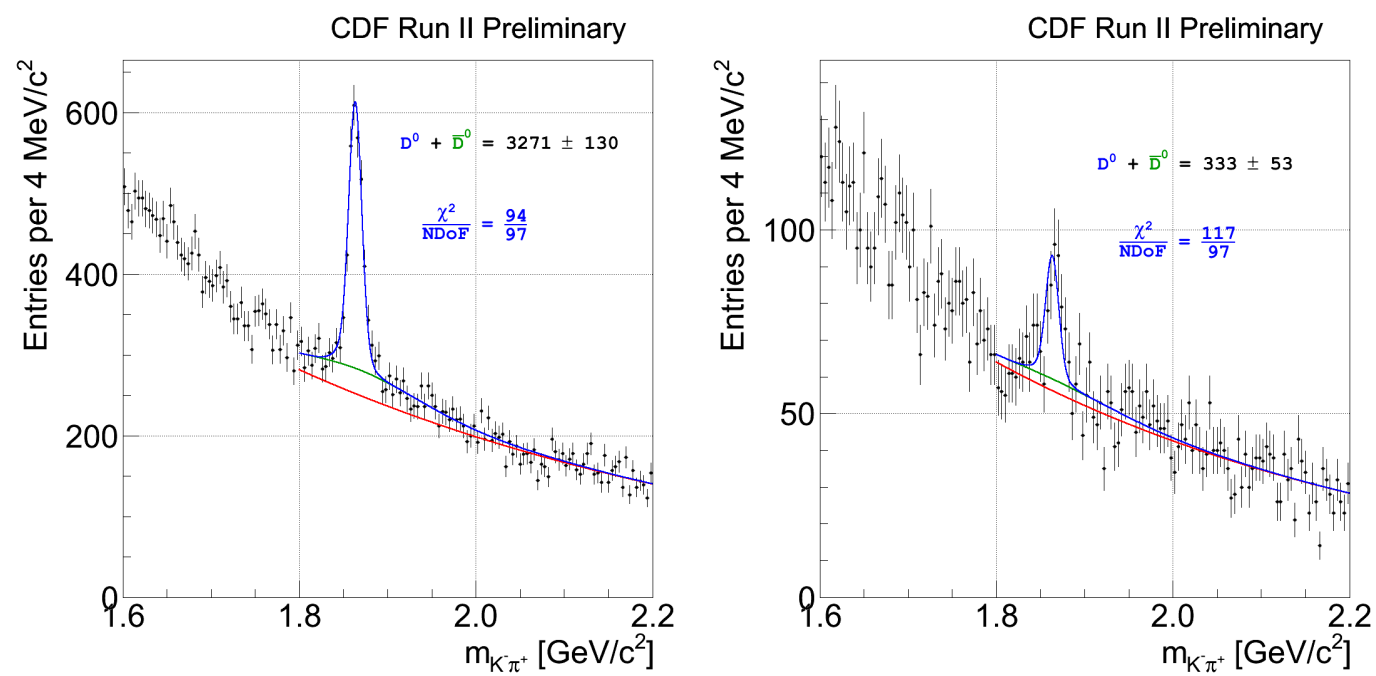

Figure 2: Invariant $K^{-} \pi^{+}$mass distribution at $\sqrt{s}=1.96 \mathrm{TeV}$ (left) and $\sqrt{s}=900 \mathrm{GeV}$ (right) of all the candidates with $p_{T} \geq 1.5 \mathrm{GeV} / \mathrm{c}$. In the fit region, stacked curves show the exponential background and broad $\bar{D}^{0}$ signal under the narrower $D^{0}$ signal resulting from the fit to the data.
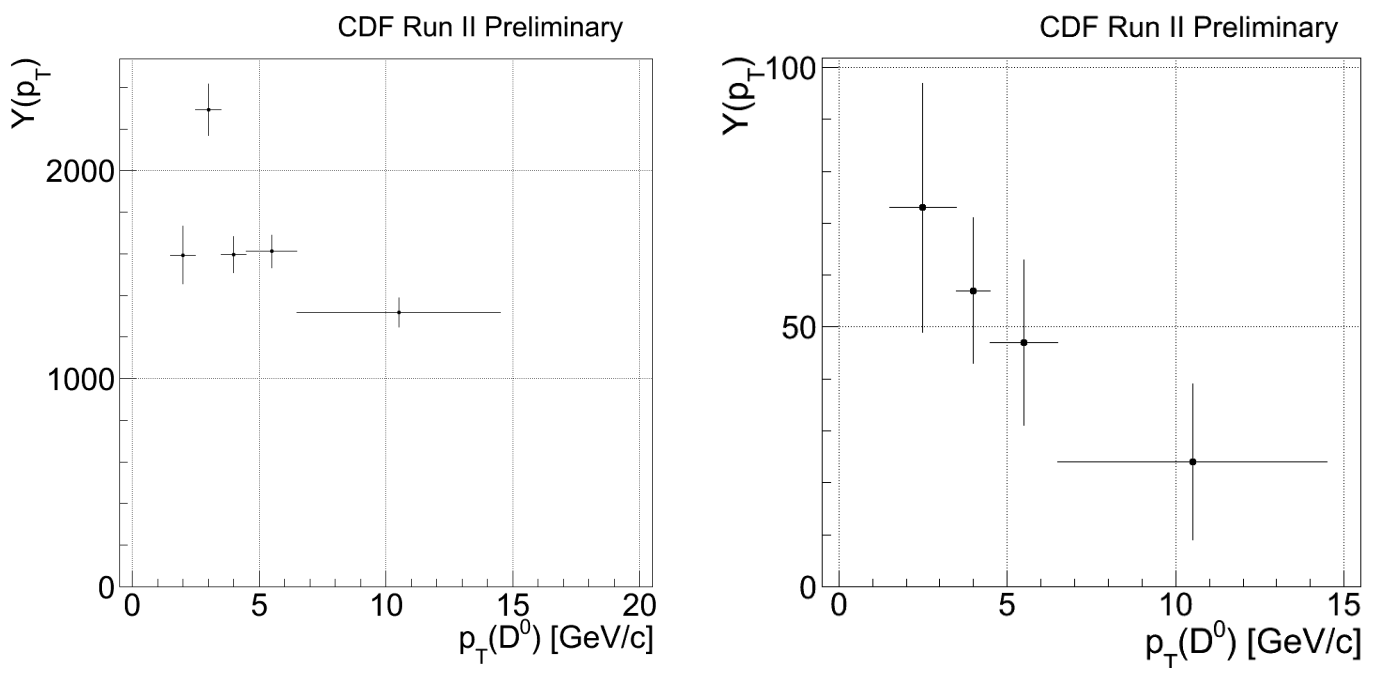

Figure 3: Measured raw yields as a function of $p_{T}$ at $\sqrt{s}=1.96 \mathrm{TeV}$ (left) and $\sqrt{s}=900 \mathrm{GeV}$ (right). They are not corrected for any acceptance effects.

We perform a binned maximum likelihood fit of the invariant $K^{-} \pi^{+}$mass. The fit function accounts for both the $D^{0}$ and $\bar{D}^{0}$ candidates. Both of them are parametrized by the sum of two Gaussians and an exponential function is used for the combinatorial background. The fit range is restricted to $[1.8 \div 2.4] \mathrm{GeV} / \mathrm{c}^{2}$ in order to reduce the (negligible) background due to other decay channels. The fit range and the signal shapes are fixed using a Monte Carlo simulation. $D^{0}$ templates have an average width of about $8 \mathrm{MeV} / \mathrm{c}^{2}$ that do not depend on the meson $p_{T} ; \bar{D}^{0}$ templates have instead a slightly greater width (that increases with the $p_{T}$ ) because of the poorer mass resolution for swapped identities of the two decay particles. 


\section{$3.2 D^{+}$}

We look for candidates fitting any possible combination of three reconstructed tracks in each event; the sum of their charges must be \pm 1 . We then assume that the two tracks with the same charge are due to pions $\left(\pi^{ \pm}\right)$and the other one to a kaon $\left(K^{\mp}\right)$. We then plot the candidate's invariant mass and we fit the signal yield. Figure 4 shows the invariant $K^{ \pm} \pi^{\mp} \pi^{\mp}$ mass distribution at $\sqrt{s}=1.96 \mathrm{TeV}$ of all the candidates with $p_{T} \geq 2.5 \mathrm{GeV} / \mathrm{c}$.

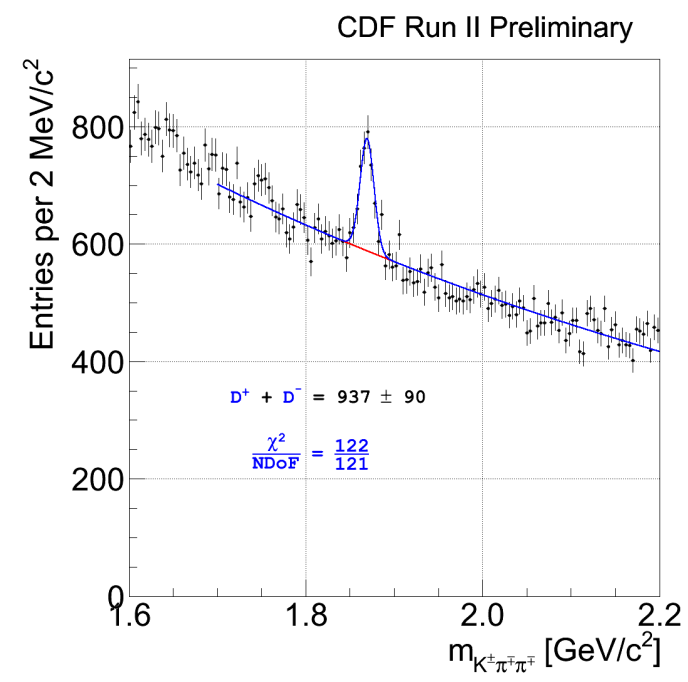

Figure 4: Invariant $K^{ \pm} \pi^{\mp} \pi^{\mp}$ mass distribution at $\sqrt{s}=1.96 \mathrm{TeV}$ of all the candidates with $p_{T} \geq 2.5 \mathrm{GeV} / \mathrm{c}$. In the fit region, stacked curves show the exponential background and the $D^{+}$signal resulting from the fit to the data.

We perform a binned maximum likelihood fit of the invariant $K^{ \pm} \pi^{\mp} \pi^{\mp}$ mass. The fit function accounts for both the $D^{+}$and $D^{-}$candidates. The signal is parametrized by a single Gaussian and an exponential function is used for the combinatorial background. The fit range is restricted to [1.7 $\div 2.0] \mathrm{GeV} / \mathrm{c}^{2}$ in order to reduce the (negligible) background due to other decay channels. The fit range and the signal shape are fixed using a Monte Carlo simulation.

\section{4. $B$ feed-down}

Our sample is also affected by a physics background due to $B$-meson decays (e.g. $B^{+} \rightarrow D^{0} X$ and $B^{0} \rightarrow D^{+} X$ ). To assess the direct fraction of candidates promptly produced in the $p \bar{p}$ collisions, we have developed a procedure that exploits the difference in the $d_{0}$ distributions between direct and secondary $D$ mesons. Because of the longer $B$ lifetime and the longer reconstructed path for the secondary mesons (it includes the path travelled by the $B$ meson), the $d_{0}$ distribution has a larger width with respect to the one related to the direct mesons. The invariant mass of the candidates is fitted as a function of the $d_{0}$. We can then fit the direct fraction from the yields plot using a Monte Carlo to fix the impact parameter distributions for the direct and secondary mesons (as shown in Figure 5 for the $D^{0}$ case). 

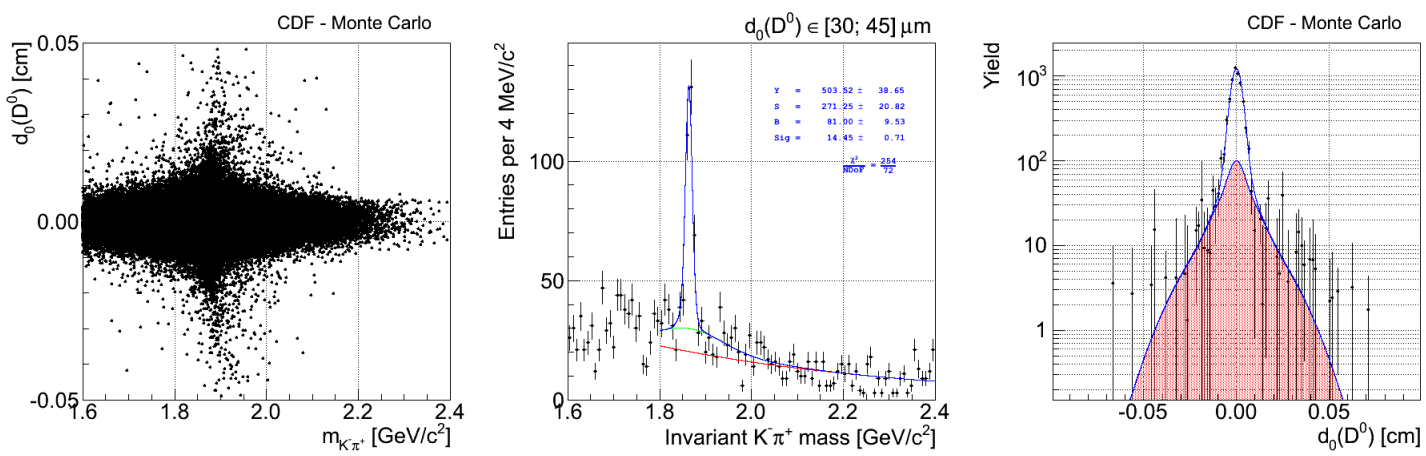

Figure 5: Impact parameter $d_{0}$ vs $K^{-} \pi^{+}$invariant mass of a $D^{0}$ Monte Carlo simulation sample (left); example of the invariant mass fit of a $d_{0}$-slice of the scatter plot (center); yields as a function of the $d_{0}$ and direct fraction fit (right).

\section{Conclusion}

We report the reconstruction of the charm signal at CDF II in the minimum-bias samples collected at two different center of mass energies $(1.96 \mathrm{TeV}$ and $900 \mathrm{GeV})$. The channels studied are $D^{0} \rightarrow K^{-} \pi^{+}$and $D^{+} \rightarrow K^{-} \pi^{+} \pi^{+}$. The total yields obtained fitting the signals of the reconstructed candidates with $p_{T} \geq 1.5 \mathrm{GeV} / \mathrm{c}$ are shown. The technique developed to assess the $B$ feed-down correction to the differential cross section is also shown.

\section{References}

[1] D. Acosta et al. (CDF Collaboration), "Measurement of Prompt Charm Meson Production Cross Sections in p $\bar{p}$ Collisions at $\sqrt{s}=1.96$ TeV”, Phys. Rev. Lett. 91, 241804 (2003).

[2] J. Beringer et al. (Particle Data Group), Phys. Rev. D 86, 010001 (2012). 